\title{
Raman evidence for nonadiabatic effects in optical phonon self-energies of transition metals
}

\author{
Yu. S. Ponosov ${ }^{1,2}$ and S. V. Streltsov ${ }^{1,2}$ \\ ${ }^{1}$ M. N. Mikheev Institute of Metal Physics UB RAS, \\ S. Kovalevskaya Str. 18, 620990 Ekaterinburg, Russia \\ ${ }^{2}$ Ural Federal University, Mira Str. 19, 620002 Ekaterinburg, Russia
}

(Dated: October 9, 2018)

\begin{abstract}
We report a Raman study of the effect of temperature on the self-energies of optical phonons in a number of transition metals with hexagonal-close-packed structure. Anisotropic softening of phonon energies and narrowing of phonon linewidths with increasing temperature are observed. These effects are reproduced in the calculations of phonon spectral functions based on $a b$ initio electronic structures and with carrier scattering by phonons taken into account. The combined observations and results of simulations indicate a relation between observed anomalies and the renormalization of the electron spectrum due to electron-phonon interaction. It is emphasized that the temperature dependence of the phonon energies resembles anharmonic behavior but is actually an electron-induced effect.
\end{abstract}

PACS numbers: 74.25.nd, 74.70.Ad, 63.20.dk, 63.20.kd

\section{INTRODUCTION}

The adiabatic approximation is commonly applied in the description of the phonon spectra of metals. Nonadiabatic (NA) corrections due to electron-phonon interaction usually are small, of the order $\sqrt{m / M}$ where $m$ and $M$ are the electronic and ionic mass, respectively ${ }^{\underline{1}}$. However, Engelsberg and Schrieffer ${ }^{2}$ were first to show that the nonadiabatic renormalization of adiabatic frequencies of long wavelength optical phonons can be larger than $\sqrt{m / M}$ if the phase velocity of phonons becomes larger than the electronic Fermi velocity $v_{f}$,

$$
\frac{\omega_{0}}{q v_{f}} \geq 1
$$

Here $q$ is the phonon wavevector and $\omega_{0}$ is the phonon frequency. Correspondingly, the electron-phonon interaction should be much enhanced for low- $q$ phonons from the NA regime. This may increase the superconducting transition temperature $T_{c}$ in correlated electron systems ${ }^{3}$. In the past years it has been proposed ${ }^{\underline{4}-6}$ that nonadiabatic effects in electron-phonon interaction could be responsible for the high-temperature superconductivity in compounds with low carrier density such as fullerenes ${ }^{7}$ or $\mathrm{Mg}_{2}{ }^{8}$. The recent measurement of Raman spectra in graphite intercalation compounds $\stackrel{9,10}{ }$ showed that the phonon frequencies related to in-plane carbon vibrations are significantly larger than those obtained from density functional theory (DFT) calculations in the adiabatic approximation. These findings have been explained by giant NA effects (up to $30 \%$ in phonon energy) calculated for a number of intercalated graphite systems and 3D metals 11 where the interband mechanism was proposed to be the main source of nonadiabaticity.

In metals, nonadiabatic effects related to the electronphonon interaction were shown to result in a singularity in the optical phonon branch for $q v_{f} \approx \omega_{0}$ which depends on the coupling constant ${ }^{12,13}$. At $q_{0}=\omega_{0} / v_{f}$ the threshold for Landau damping for an optical phonon has been predicted and the temperature behavior of the phonon frequencies and linewidths has been discussed ${ }^{13-15}$.

In contrast to neutron inelastic scattering, Raman spectroscopy only covers the near-zone-center regime $\left(10^{5}-10^{6} \mathrm{~cm}^{-1}\right)$ but offers high resolution. The experimental detection of NA effects in metals, however, is rather difficult because of smearing of the probed wavevector. It results from the finite penetration depth of the laser light and leads to the observation of both adiabatic and NA contributions to optical phonon frequencies and linewidths. Such effects of spatial dispersion on the electron-phonon interaction were first found in pure hexagonal-close-packed (hcp) metals with long mean free path $^{16}$. There, the coupling is indicated by an anomalous temperature behavior of the $E_{2 g}$ phonon linewidths. Along with this anomaly, the phonon frequencies showed a typical softening upon cooling ${ }^{17-19}$.

In this report we present the results of new experimental studies of NA effects in a number of hcp transition metals. We confirm that in these anisotropic metals the NA effects lead to a variation of optical phonon energy being dependent both on the value and direction of the phonon momenta ${ }^{20}$. We simulated the spatial dispersion effects and the anomalous temperature behavior of the phonon linewidth in our model calculations. They are based on density functional theory (DFT) electronic structures and take into account intraband transitions. It is shown that renormalization of the bare electron spectrum due to electron-phonon interaction is the main mechanism of phonon self-energy changes. In particular, it is specified that the phonon softening with increasing temperature is not the usual anharmonic effect but is due to the electron-phonon interaction. 


\section{EXPERIMENTS}

For temperature-dependent measurements, electropolished plates of $\mathrm{Y}, \mathrm{Zr}, \mathrm{Ti}, \mathrm{Ru}$, and Os single crystals (residual resistance ratio $\geq 50$ ) with different orientations of the scattering surface were placed into an optical cryostat (Oxford or Cryovac). Most measurements were performed with excitation by laser radiation of wavelengths $514 \mathrm{~nm}$ and $633 \mathrm{~nm}$. The focal spot on the sample varied from 2 to $10 \mu \mathrm{m}$ diameter, depending on settings. For $\mathrm{Ru}$ and $\mathrm{Os}$, additional lines of a $\mathrm{Kr}$ ion laser were used. Raman spectra were recorded by Renishaw and Labram microscope spectrometers with the spectral resolution (approximated by a Gaussian shape) of about $3 \mathrm{~cm}^{-1}$. In order to obtain the intrinsic phonon frequencies and linewidths, the measured spectra were fitted with a Voigt expression.

\section{CALCULATIONS}

The Raman line shape in metals is affected by both anharmonicity and electron-phonon coupling. Calculations of the phonon spectral function were carried out in order to theoretically estimate the effects of electron-phonon interaction on the phonon energies and linewidths of the investigated metals. As a result of the wavevector smearing of the light field in a metal and in the presence of strong dispersion of the frequency and damping, a measured phonon line shape may have a rather complicated form. The phonon spectral function $I(\omega)$ was calculated by taking into account the frequency and temperature dependences of the phonon self-energies $\Pi\left(q, \omega, \omega_{0}\right)$ :

$$
\begin{aligned}
& I(\omega)=\frac{4 \omega_{0}^{2}}{\pi} \times \\
& \int_{0}^{\infty} d q \frac{U(q) \Gamma\left(q, \omega, \omega_{0}\right)}{\left[\omega^{2}-\omega_{0}^{2}-2 \omega_{0} \Pi^{\prime}\left(q, \omega, \omega_{0}\right)\right]^{2}+4 \omega_{0}^{2} \Gamma^{2}\left(q, \omega, \omega_{0}\right)}
\end{aligned}
$$

Here $\omega_{0}$ is the bare (uncoupled) phonon energy, $\Gamma\left(\vec{q}, \omega, \omega_{0}\right)=\Gamma_{0}+\Pi^{\prime \prime}(\vec{q}, \omega)$ is the total width, $\Pi^{\prime}(\vec{q}, \omega)$ and $\Pi^{\prime \prime}(\vec{q}, \omega)$ are the real and imaginary parts of the phonon self-energy. The bare line width $\Gamma_{0}$ includes zerotemperature contributions from defects, anharmonicity, etc. The breakdown of optical wavevector conservation in a metal leads to coupling of the light to phonons with a spread of wavevectors and hence of frequencies. Following 22 we take $U(\mathrm{q})$ to have the form $U(q) \propto$ $4 q^{2} /\left|q^{2}-\xi^{2}\right|^{2}$, where $\xi=\xi_{1}-i \xi_{2}=\left(2 \omega_{i} / c\right) \times(n-i k)$. For incident laser energy $\omega_{i}$ probed wavevector distribution $U(q)$ respresents a skew lineshape with a peak position $q_{i}=\left(2 \omega_{i} / c\right) \times \sqrt{n^{2}+k^{2}}$ and was estimated on the basis of experimental refraction index $\mathrm{n}$ and extinction coefficient $\mathrm{k}$ for investigated metals 23,24 .

The phonon self-energy originates from intraband electronic transitions in our simulation. We used $\Pi(q, \omega)$ in the form: ${ }^{13}$

$$
\begin{aligned}
& \Pi\left(q, \omega, \omega_{0}\right)=\oint \frac{d s_{f}}{v_{f}} g^{2}\left(k_{f}, q, \omega_{0}\right) \times \\
& \left\{\left(\int_{-\infty}^{\infty} d \varepsilon \frac{f(\varepsilon)-f(\varepsilon+\omega)}{\omega-q v_{f}^{z}-\Sigma^{A}(\varepsilon)+\Sigma^{R}(\varepsilon+\omega)}\right)-2\right\} .
\end{aligned}
$$

Here, $d s_{f}$ is the area element of the Fermi surface, $v_{f}$ - the electron velocity, $g$ - the matrix element of the electronphonon interaction, $f(\varepsilon)$ is the Fermi function, and $z$ denotes the normal to the sample surface. The advanced and retarded quasi-particle electronic self-energies $\Sigma^{A}(\epsilon)$ and $\Sigma^{R}(\epsilon+\omega)$ determine the electron spectrum renormalization near the Fermi level due to different interactions. In the case of electron-phonon scattering, the real and imaginary parts of $\Sigma(\epsilon) \operatorname{are}^{25}$

$$
\begin{aligned}
& \Sigma^{\prime}(\epsilon)=\int d \Omega \alpha^{2} F(\Omega) \times \\
& \Re\left[\psi\left(\frac{1}{2}+i \frac{\epsilon+\Omega}{2 T}\right)-\psi\left(\frac{1}{2}+i \frac{\epsilon-\Omega}{2 T}\right)\right], \\
& \Sigma^{\prime \prime}(\epsilon)=\pi \int d \Omega \alpha^{2} F(\Omega) \times \\
& {\left[2 n_{B}(\Omega)-f(\epsilon-\Omega)+f(\epsilon+\Omega)+1\right]+\nu .}
\end{aligned}
$$

Here $\nu$ is the electron-impurity scattering rate, $\Psi$ the digamma function, $\Omega$ the phonon energy, $\alpha^{2} F(\Omega)$ the Eliashberg spectral function for the electron-phonon interaction, and $n_{\mathrm{B}}$ the Bose function. The phonon densities of states $F(\Omega)$ from Refs ${ }^{26,27}$ or from measured second order Raman spectra (in case of Os, see Fig.S1) were used in our calculations.

The electron velocities on the Fermi surface have been obtained from band-structure calculations that use the linearized muffin-tin orbital method (TB-LMTOASA ${ }^{28}$ ) in the local density approximation. The obtained Fermi surfaces are in agreement with those calculated earlier ${ }^{29}$. Integration over the Fermi surface was performed with a fine mesh of $125000 \mathrm{k}$-points in the full Brillouin zone.

\section{RESULTS}

\section{A. Group III metal yttrium}

Figure 1 shows measured and calculated lineshapes and Fig. 2 present the energy and width of the $E_{2 g}$ optical mode in yttrium as a function of temperature. The phonon frequencies for probed $q$ directions, cf. Fig. 1 and Fig. 2, exhibit $\mathrm{a} \simeq 5 \%$ difference at low temperatures. The difference almost disappears at room temperature. Low-temperature linewidths, cf. Fig. 1 and Fig. 2, are also different and show non-monotonic temperature behavior with maximum near $100 \mathrm{~K}$ for $\mathrm{q} \|[00 \xi]$, then decreasing with further increase of temperature. There are 


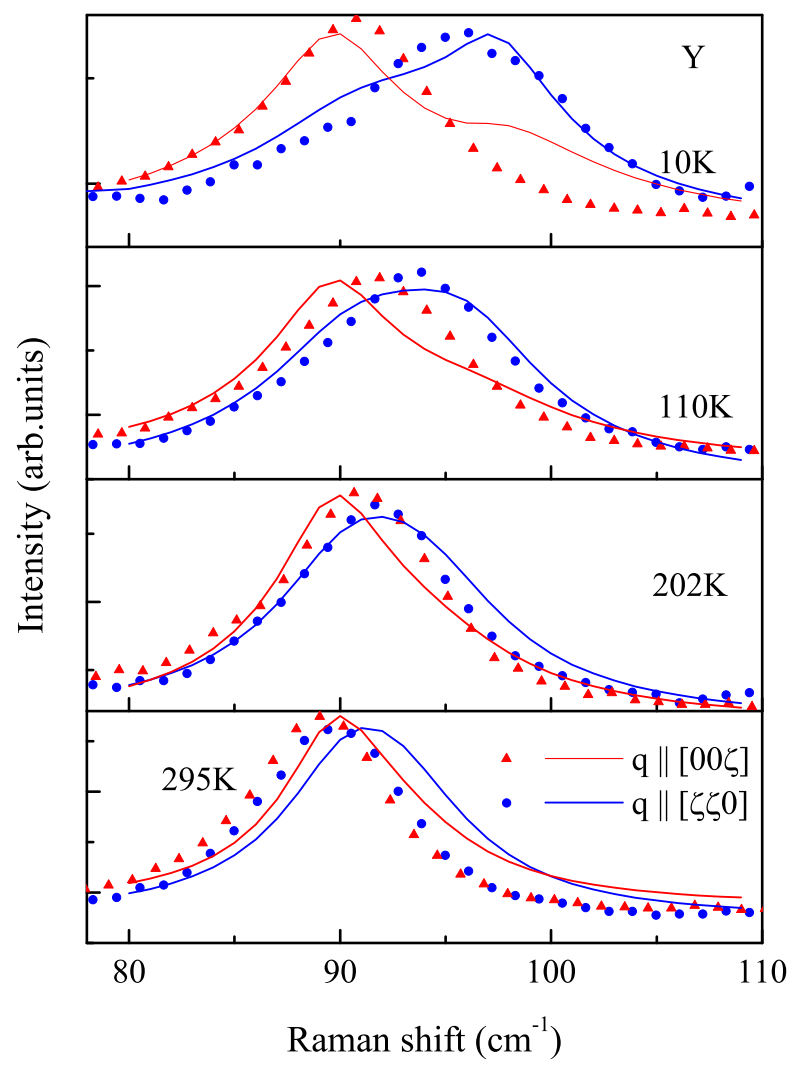

FIG. 1. Measured (symbols) and calculated (lines) phonon spectral functions of yttrium at different temperatures for two directions of the wavevector $q$. The excitation wavelength was $633 \mathrm{~nm}$.

no low-temperature phase transitions in Y. It is therefore natural to assume that the observed anisotropy of the phonon frequencies and widths and unusual temperature dependence of the latter is due to the electron-phonon interaction.

Using Eqs. 2 - 5 we performed calculations of phonon line shapes for different wavevector directions and temperatures. In these and the following calculations we used values of $\Gamma_{0}=0.5-0.7 \mathrm{~cm}^{-1}$ and $\nu=10 \mathrm{~cm}^{-1}$. Adjusted parameters were $\omega_{0}, g$ and an electron-phonon coupling constant $\lambda=2 \int d \Omega \alpha^{2} F(\Omega) / \Omega$. Because the phonon energies in all the studied metals do not depend on the $q$ direction at room temperature, this allows to vary the bare frequency $\omega_{0}$ and the matrix element of electronphonon interaction $g$ in narrow limits. Using literature values of $\lambda_{t r}=0.62^{30}$ or calculated $\lambda=0.55^{31}$ couldn't provide any acceptable agreement with experimental results. The best agreement was obtained using $\lambda=0.13$. Parameters used in the calculations for the five metals are listed in Table $\amalg$

The $q$-dependences of the calculated phonon selfenergies are shown in Fig. 3 together with probed wavevector distribution. It follows from Fig. 3] that the calculated $q_{0}$ value for the Landau damping threshold and strong dispersion region for $\mathrm{q} \|[00 \xi]$ is approximately
TABLE I. Parameters used in calculations of phonon selfenergies of the investigated metals.

\begin{tabular}{llllll}
\hline \hline & $\omega_{0}\left(\mathrm{~cm}^{-1}\right)$ & $g\left(\mathrm{~cm}^{-1}\right)$ & $\Gamma_{0}\left(\mathrm{~cm}^{-1}\right)$ & $\lambda$ & $\lambda_{E_{g}}$ \\
$\mathrm{Y}$ & 97 & 95 & 0.5 & 0.13 & 0.056 \\
$\mathrm{Zr}$ & 104 & 145 & 0.5 & 0.49 & 0.059 \\
$\mathrm{Ti}$ & 157 & 203 & 0.7 & 0.54 & 0.064 \\
$\mathrm{Ru}$ & 213 & 200 & 0.5 & 0.66 & 0.042 \\
$\mathrm{Os}$ & 179 & 213 & 0.5 & 0.72 & 0.042 \\
\hline \hline
\end{tabular}

two times less than for the in-plane $q$ direction. The value of $q_{0}$ was evaluated at bare phonon frequency $\omega_{0}$ (Table from Eq.3 where we used isotropic electronic self-energies $\Sigma$. Therefore, the low-temperature difference of $q_{0}$ and phonon energies for different q-directions is due to the difference of average bare velocities $v_{f}^{z}$. In its turn, the latter are determined by both a bare electron velocity and a shape of the Fermi surface, which sets the value of the average velocity for a certain $q$ - direction. The result shown for yttrium in Fig. 3 is commented in Supplemental information (see Fig.S2).

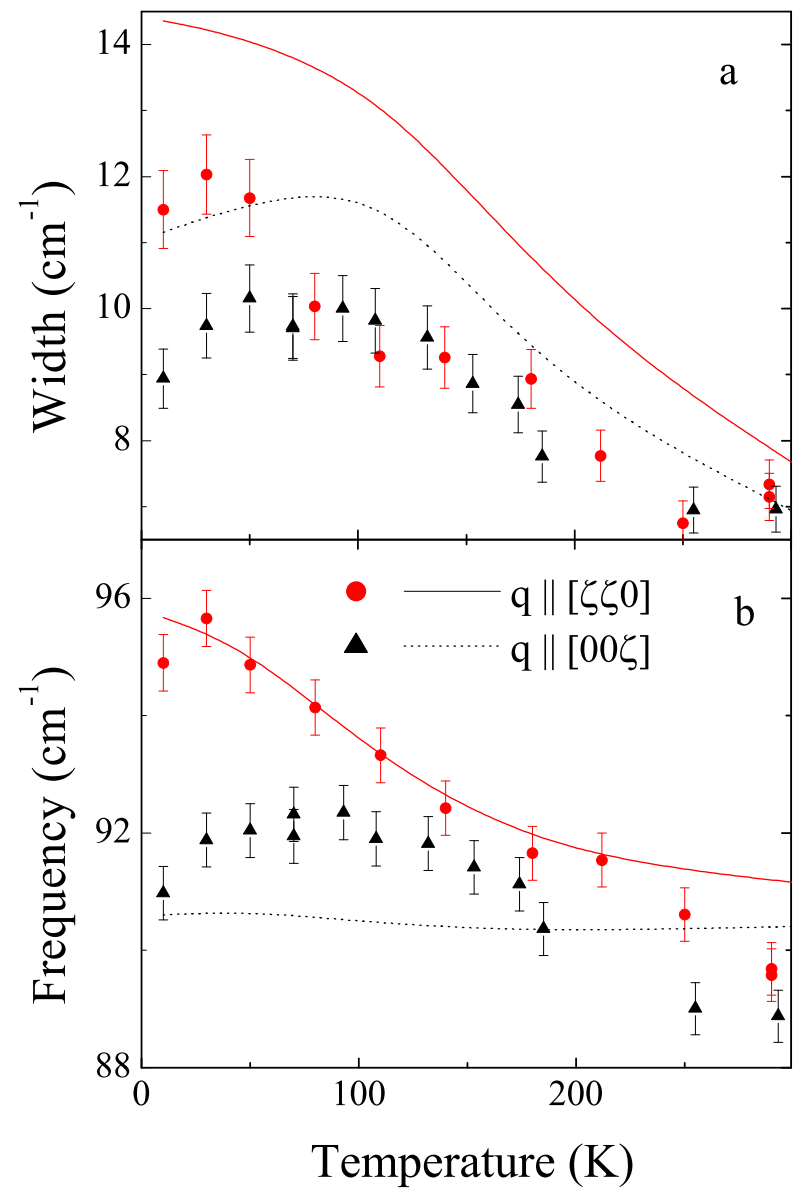

FIG. 2. Experimental (symbols) and calculated (lines) temperature dependence of the phonon linewidth (a) and frequency (b) in yttrium for two wavevector directions. The excitation wavelength was $633 \mathrm{~nm}$. 
Therefore, having in mind the wavevector distribution, it becomes clear that for $\mathrm{q} \|[00 \xi]$ the adiabatic region provides the main contribution to the phonon frequency. For the in-plane $q$ direction the contribution of the NA region prevails, where the phonon frequencies are higher at low temperatures. Strong renormalization of the electron mass (velocity) in the NA region 13 leads to the phonon frequency decrease when temperature increases. Although such temperature trend is qualitatively similar to anharmonic behavior, the actual reason for phonon softening is a disappearance of NA contribution at high temperatures. The anomalous narrowing of the phonon linewidth with increasing temperature can be attributed to a decrease of the number of electron states which strongly interact with phonon and provide its damping both in adiabatic and nonadiabatic q-regions. The electron lifetime in the NA region decreases due to an electron-phonon scattering which leads to the increase of the phonon width at intermediate temperatures $11,13,14$. The interplay between these opposite trends is manifested sometimes in the non-monotonic dependences of the phonon widths.

The solid lines in Fig. 2(a,b) show the frequencies and widths obtained from fitting of the phonon spectral functions calculated using phonon self-energies of Fig. 3. The agreement with experimentally observed tendencies is rather satisfactory though our simple calculation uses constant and isotropic $\alpha^{2}(\Omega)$ and $g$. It should be noted that phonon spectral functions calculated for low temperatures have rather complicated shapes because of wavevector smearing. They show shoulders originating from NA region of the spectrum (for q $\|[00 \xi]$ ), when the main contribution is determined by the adiabatic region or, conversely, for the in-plane $q$ direction, when the peak from NA region dominates (Fig. 1). Experiment actually finds asymmetric line shapes but the presence of the second peak is less pronounced in most cases (Fig. 10. As a rule, phonon peaks become symmetric at high temperatures. We have not tried to decompose the experimental and calculated peaks for the components because the resulting form is a complex integral function of the probed distribution of the wavevectors and anomalous phonon dispersion. Instead, both the experimental and calculated line shapes were fitted by a Voigt (Lorentz) distribution to determine the central frequency and linewidth. Unfortunately, the use of such a procedure for fitting the calculated spectral functions sometimes leads to frequency shifts and band broadening due to the appearance of double peaks.

It should be noted that little information on the $\lambda$ magnitude of $\mathrm{Y}$ exists. Our estimate, indicating weak electron-phonon interaction in spite of high density of states at the Fermi level, is in agreement with absence of superconductivity in $\mathrm{Y}$ at atmospheric pressure. Estimated mode constant $\lambda_{E_{2 g}}=g^{2} N_{F} / \omega_{0} \simeq 0.056$, where $N_{F}$ is a total density of states at the Fermi level.

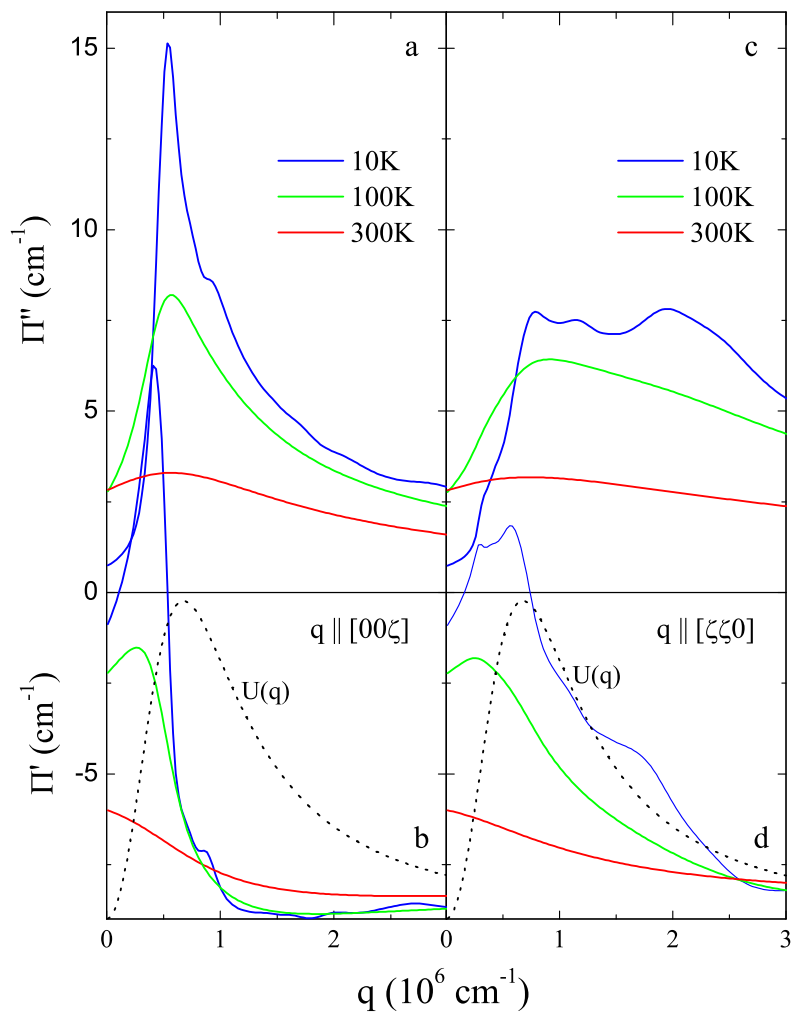

FIG. 3. Calculated imaginary $(\mathrm{a}, \mathrm{c})$ and real $(\mathrm{b}, \mathrm{d})$ parts of the phonon self-energy of $\mathrm{Y}$ versus wavevector $q$ for two qdirections and different temperatures. Dotted line in (b) and (d) shows probed wavevector distribution for excitation wavelength $633 \mathrm{~nm}$.

\section{B. Group IV metals -zirconium and titanium}

The measured phonon lineshapes in the group IV metals $\mathrm{Zr}$ and $\mathrm{Ti}$ are shown in Fig.S3. The temperature dependences of the $E_{2 g}$ frequencies and widths in $\mathrm{Zr}$ and $\mathrm{Ti}$ are presented in Fig. 4. The results for $\mathrm{Zr}$ are similar to those for $\mathrm{Y}$ and show differences in frequencies and widths for in-plane and $c$-axis $q$ directions at temperatures $\triangleleft 100 \mathrm{~K}$ (Fig.4). Our calculations reproduce the tendency of anisotropic phonon frequency softening and width narrowing with increasing temperature. The calculated linewidth for momentum along an $c$ axis at low temperatures exceeds the linewidth for the momentum in the basal plane in contrast to the experiment (Fig. 4(a,b)). The reason is an appearance of a shoulder on the high frequency side of the phonon peak. This leads to upward shift of the frequency and peak broadening with accepted procedure for fitting of calculated spectral function.

Another situation is observed in $\mathrm{Ti}$ where the phonon parameters were found to be isotropic for all three $q$ directions. In Fig. 4(c,d) we present results for q \| $[00 \xi]$ and $\mathrm{q} \|[\xi \xi 0]$ in addition to earlier data for $\mathrm{q} \|$ $[\xi 00]^{19}$. The calculations of the phonon self-energies in 


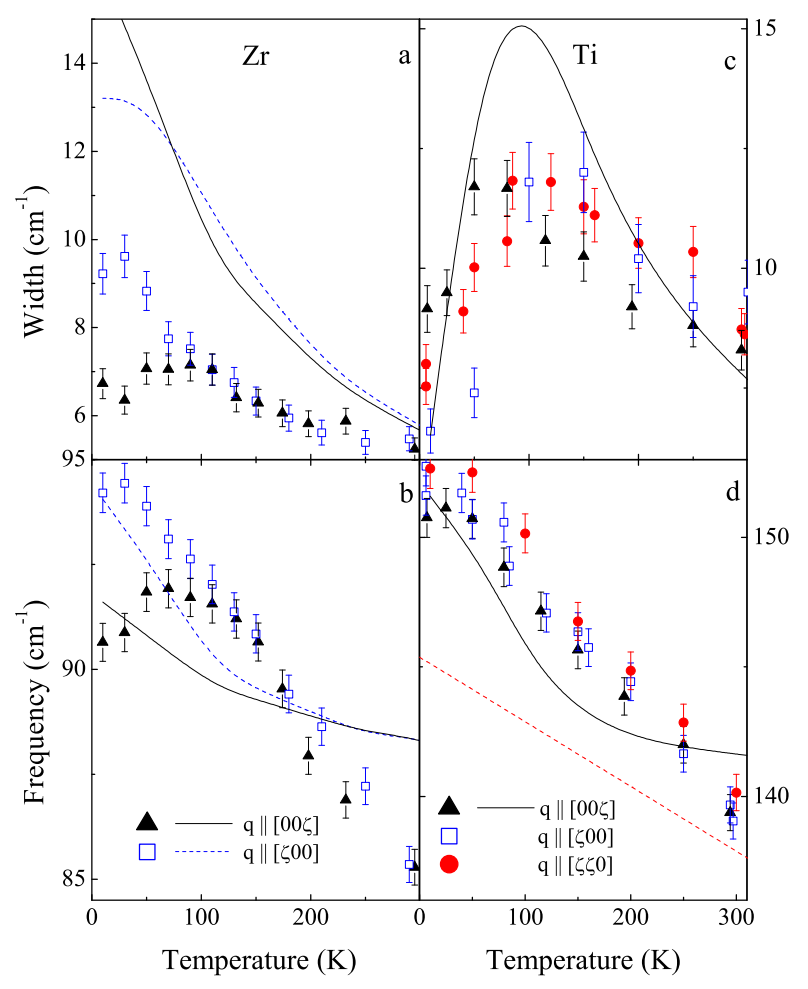

FIG. 4. Experimental (symbols) and calculated (lines) temperature dependence of the phonon linewidth and frequency in zirconium $(\mathrm{a}, \mathrm{b})$ and titanium $(\mathrm{c}, \mathrm{d})$ for different directions of the wavevector $q$. The excitation wavelength was $633 \mathrm{~nm}$. Dashed line in (d) is interpolation of high-temperature neutron data ${ }^{32}$ for $E_{2 g}$ mode in Ti.

Ti for bare phonon frequency $\omega_{0}$ (Table \) show that in this case probed momenta distribution located mainly in the NA region (Fig. 5). This is the reason of rather strong $(\simeq 8 \%)$ frequency softening and non-monotonic behavior of width (see temperature dependence of $\Pi^{\prime \prime}$ in Fig. 51) when temperature increases. Interpolation of high-temperature neutron data ${ }^{32}$ (Fig. 4(d)) shows strong deviation from Raman phonon frequencies at low temperatures. Calculations for different $q$ directions gave almost the same results (solid lines in Fig. 4(c,d)) which describe experimental phonon frequencies and widths rather good. Nevertheless, found in experiment differences in low -temperature widths suggest contribution of the intraband effects. It is interesting that comparable quantitative NA effect on the phonon frequency in Ti was calculated in Ref ${ }^{11}$, but the main source of such phonon renormalization was attributed to interband transitions.

\section{Group VI metals - ruthenium and osmium}

Figures [6, 7]and 8] show the temperature dependences of the spectra, phonon frequencies and widths for $4 \mathrm{~d}$ and $5 \mathrm{~d}$ metals of VI group $\mathrm{Ru}$ and Os. Calculated electronic structures and Fermi surfaces for both metals are very

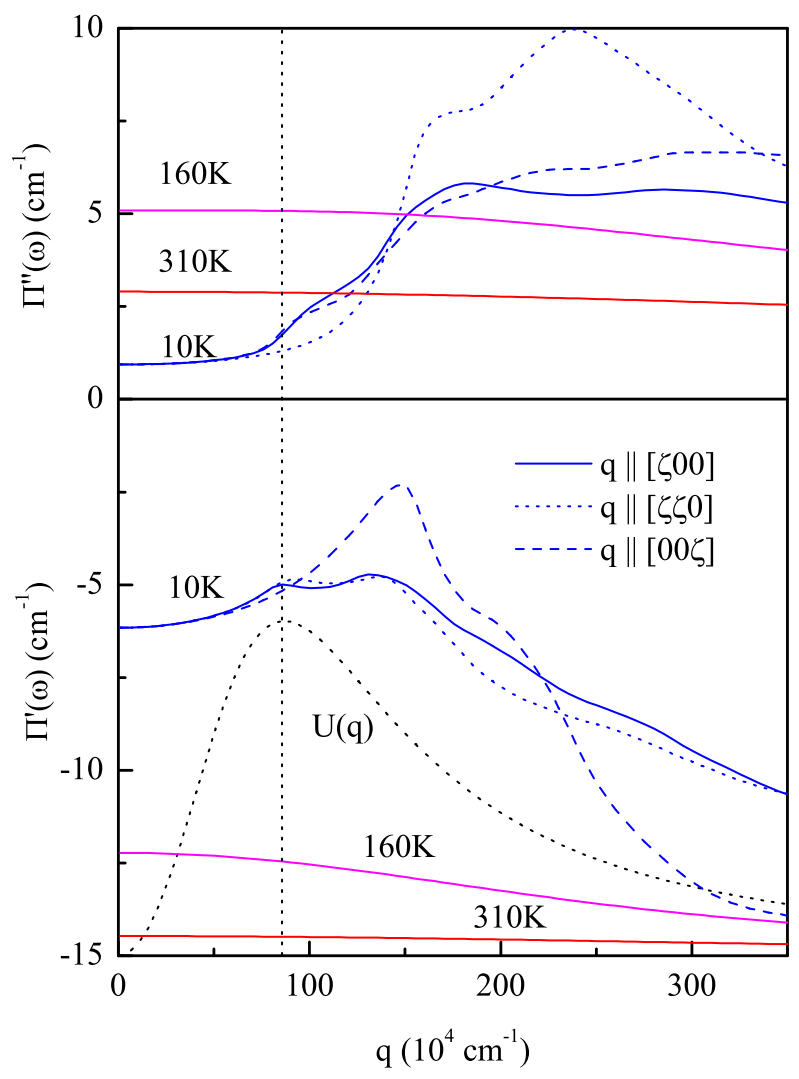

FIG. 5. Calculated real (a) and imaginary (b) parts of the phonon self-energy of Ti versus wavevector $q$ for different qdirections and temperatures. Dotted line in (b) and (d) shows probed wavevector distribution for excitation wavelength 633 nm.

similar. Therefore it is not surprising that we found similar temperature behavior of their phonon self-energies. In ruthenium NA effects in electron-phonon interaction are observed through the differences of the phonon selfenergies for different wavevector directions. We found that at low-temperature the frequencies of $\mathrm{E}_{2 g}$ phonon for q $\|[00 \xi]$ are by $4-5 \mathrm{~cm}^{-1}$ less than for in-plane momentum directions, but they become almost equal at room temperature (Fig.S4 and Fig. 6). The frequencies decrease with increasing temperature for all momentum directions, while the widths sometimes show a nonmonotonic dependence, especially for $\mathrm{q} \|[\xi \xi 0]$, where the low-temperature width is close in magnitude to the one observed at $300 \mathrm{~K}$. Calculated phonon frequencies and widths, also shown in Fig. 6] satisfactorily reproduce the anisotropy in the temperature dependence of frequencies, but show monotonic increase for all q-directions in the contrast experimental data for q $\|[\xi \xi 0]$. For this direction both the adiabatic and NA regions contribute to peak intensity that leads to large growth of the linewidth at low temperatures (Fig.S4 and Fig. 6).

The variation of osmium refraction index $\mathrm{n}$ and extinction coefficient $\mathrm{k}$ with wavelength allows the wavevector $\mathrm{q}$ to be tuned in the region $(6-18) \cdot 10^{5} \mathrm{~cm}^{-1}$. Such vari- 


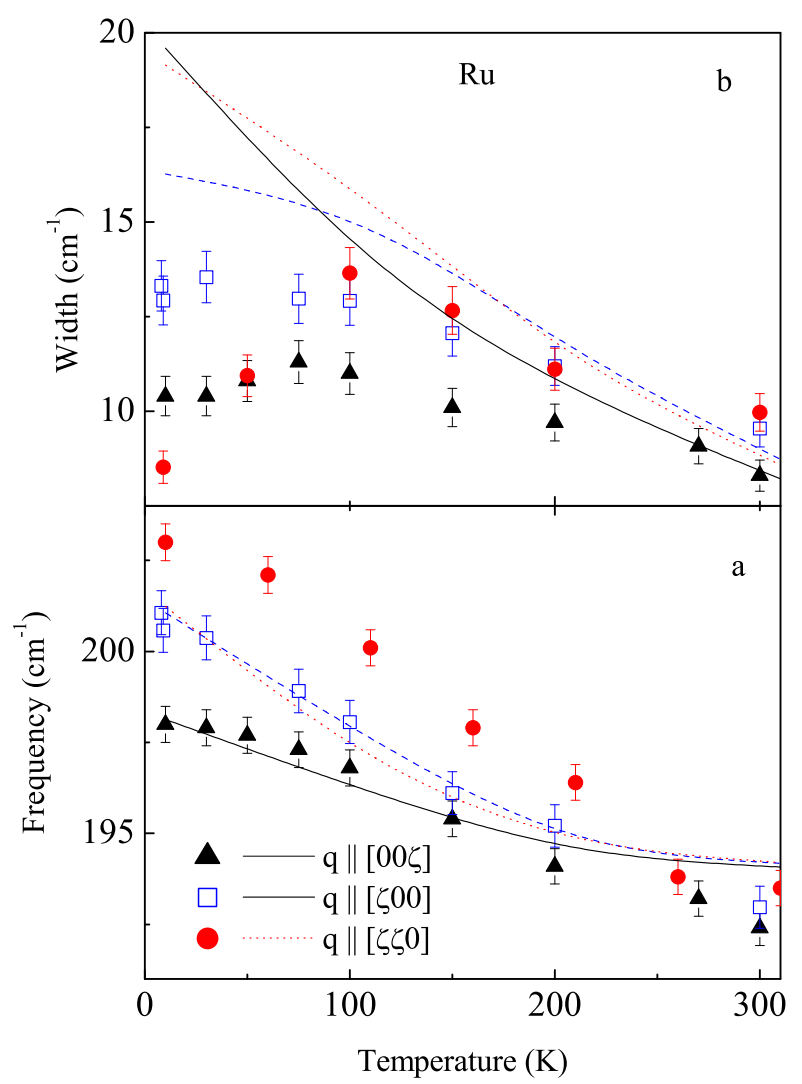

FIG. 6. Experimental (symbols) and calculated (lines) temperature dependence of the phonon frequency (a) and linewidth (b) in ruthenium for different directions of the wavevector $q$. The excitation wavelength was $647 \mathrm{~nm}$.

ation leads to the changes in the frequencies and widths of the phonon lines for certain directions of wavevector $^{20}$ (Fig. 7 and 8). Particularly noticeable the changes in low-temperature frequencies for directions of q $\|[00 \xi]$ and $\mathrm{q} \|[\xi 00]$. They increase during the transition to the longer-wavelength excitation and a corresponding decrease in the probed wavevector (see inset in Fig. 8). Phonon lines in Os Raman spectra are superimposed on broad electronic background ${ }^{20}$, which is rather structureless for $514 \mathrm{~nm}$ excitation but show very expressed profile for red line excitation at 647 and $676 \mathrm{~nm}$ (Fig. 7). The maximum of this intraband continuum is close to the energy of $E_{2 g}$ phonon and its temperature behavior is related to the phonon self-energy trend. It is not surprising because the frequency and temperature dependence of inelastic light scattering by electronic excitations is determined by imaginary part of Eq.3 where the matrix element of electron-phonon interaction $g$ is replaced by matrix element of electron-photon interaction ${ }^{33}$. As with other investigated metals, the anomalous broadening of the phonon lines upon cooling was observed in osmium. A pronounced maximum at $\mathrm{T} \approx 100 \mathrm{~K}$ was found for direction q $\|[\xi \xi 0]$, where the low-temperature linewidth was close to its room temperature value for $647 \mathrm{~nm}$ exci- tation, as in the case of ruthenium (Fig. 6). As one can see, the intensity of electronic continuum for this direction also shows nonmonotonic behavior in this temperature range. This similarity confirms that intraband electronic excitations determine the phonon self-energy. The general trend found is an increase in the low-temperature frequencies and widths of the phonon lines with decreasing wavevector probed. This clearly indicates the growing contribution of NA region of wavevectors to the measured phonon frequencies and widths. We performed calculations of the phonon spectral functions at different temperatures (Eqs. 2015) for both excitation wavelengths. The results obtained (Fig. 8) reproduce observed at low temperatures phonon softening and line narrowing, when the probed momentum increases. However, it is not possible to describe a line narrowing at low temperatures

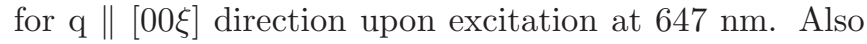
the low temperature linewidths for excitation at $514 \mathrm{~nm}$ are overestimated due to arising high energy shoulders in calculated spectral functions.

\section{DISCUSSION}

Thus, the narrowing of the phonon lines or nonmonotonic behavior with increasing temperature is observed for all investigated metals. Typically, the phonon frequencies decrease with increasing temperature. Temperature behavior of the phonon parameters depends on the direction of probed wave vector which. The lowest anisotropy was found in titanium, where the frequencies for q-directions practically coincide. Found q - dependences imply the contribution of the intraband electronic transitions to the observed effects. The proposed explanation of these effects is based on the account of electron spectrum renormalization due to carrier scattering by phonons.

The main contribution to the narrowing of the phonon lines is due to the increase of the electron relaxation rate with increasing temperature. This leads to a decrease the

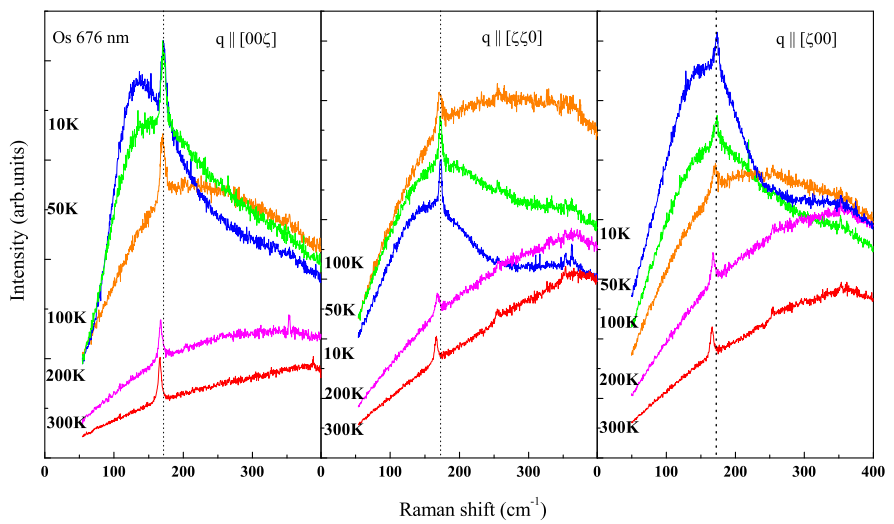

FIG. 7. Raman spectra in osmium measured at different temperatures for different q-directions. Excitation was $676 \mathrm{~nm}$. 


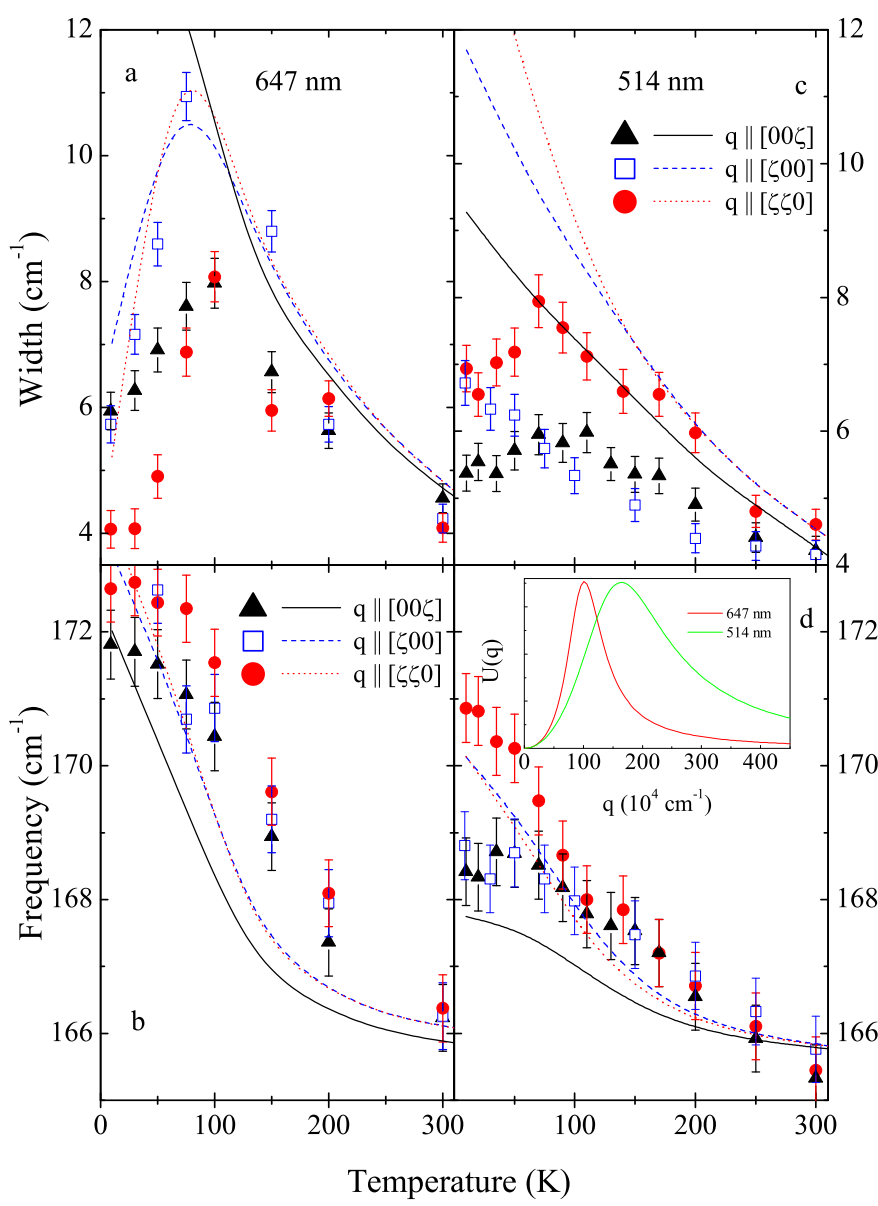

FIG. 8. Experimental (symbols) and calculated (lines) temperature dependence of the phonon frequency and linewidth in osmium for two excitation energies and for different directions of the wavevector. The inset in (d) shows phonon momentum distributions for the two excitation energies.

time during which the electron moves in phase with the phonon and interacts effectively with it in the adiabatic region of the spectrum. Otherwise, in the NA region the phonon damping increases at intermediate temperatures due to a decrease of the electron lifetime. Interplay of these mechanisms sometimes results in a non-monotonic temperature behavior of the total phonon widths.

Possible reasons for discrepancies between the experimental and calculated results may be inaccuracies in the calculations of the Fermi surface shape and the distribution of electron velocities on it. In addition, a rough calculation using the isotropic renormalization of the electron spectrum and the same matrix elements of electronphonon interaction for all Fermi surface sheets, may not produce the subtle features in the behavior of the phonon self-energies. Nevertheless, overall decrease of the frequencies and linewidths upon transition to shorter excitation wavelength in Os was reproduced in calculations.

There are two additional unaccounted effects which could distort the calculated spectral functions for all met- als. One of them is the neglecting of the influence of screening in the case of longitudinal phonons for the momentum direction along $[\xi \xi 0]$. The point group $D_{6 h}$ of a hcp crystal leads to first-order Raman activity for only one doubly degenerate zone-center optical phonon mode of $E_{2 g}$ symmetry. In this vibration, two sublattices undergo opposite displacements in the $x$ - and $y$-directions of the basal plane. For small finite wavevector along the $[00 \xi]$ direction (measurements from (0001) surface) the doubly degenerate transverse phonon is probed, while the longitudinal mode in $[\xi \xi 0]$ direction and the transverse mode in [ $[00]$ direction may be studied in measurements from (1010) and (1210) planes. It is possible that the difference in low-temperature phonon frequencies for different $\mathrm{q}$ in the basal plane in Os and Ru (see Figs.6-8) is associated with this effect. The second unaccounted effect is associated with a possible line shape change of the phonon lines due to the Fano interference with the electronic continuum.

In all performed calculations we did not take into account another possible sources of phonon softening with increasing temperature which are thermal expansion and higher anharmonic processes. Estimates made show the thermal expansion effects do not exceed $10 \%$ of the observed phonon softening in all investigated metals. Some anharmonic contributions to the phonon damping and energy were observed in $\mathrm{Ti}$ at $T \geq 300 \mathrm{~K}$; no linewidth broadening was found in $\mathrm{Ru}$ and $\mathrm{Os}$ up to $800 \mathrm{~K}$. Therefore, we believe that the main part of the temperature changes in phonon self-energies are associated with electronic contributions.

\section{CONCLUSIONS}

We present new results on the temperature and momentum dependent optical $E_{2 g}$ phonon self-energies in five hcp transition metals. The comparison of experimental and calculated phonon spectral functions clearly evidences that the nonadiabatic effects in electron-phonon interaction strongly contribute to detected anomalies. According to our calculations, the main part of the effects comes from the intraband mechanism. The contribution of the interband transitions still needs to be estimated. Despite differences of experimental and calculated phonon parameters, we were able qualitatively (and sometimes even quantitatively) to describe the observed temperature behavior of the self-energies of the long wavelength optical phonons and their anisotropy for all investigated metals. Though these effects amount to a few percent, however, they determine the temperature dependence of the phonon frequencies, which can be mistakenly identified with the anharmonic behavior.

As a general remark, the ability of a Raman experiment to probe the nonadiabatic effects on the optical phonon self-energy requires a careful analysis of temperature dependence of phonon self-energies in metals and doped semiconductors to separate the effects of phonon- 
phonon and electron-phonon interactions.

\section{ACKNOWLEDGMENTS}

We thank K. Syassen for valuable discussions. The research was supported by the grant of the Russian Sci- entific Foundation (project no. 14-22-00004).
1 A. B. Migdal, Sov.Phys. JETP 7, 996 (1958).

2 S. Engelsberg and J. R. Schieffer, Phys. Rev. 131, 993 (1963).

3 E. G. Maksimov, M. L. Kulic, and O. V. Dolgov, Advances in Condensed Matter Physics 2010, ID 423725 (2010).

4 E. Cappelluti, and L. Pietronero, Phys. Stat. Sol. (b) 242, 133 (2005).

5 A. S. Alexandrov and V. V. Kabanov, Phys. Rev. B 54, 3655 (1996)

6 Matteo Calandra, Michele Lazzeri, Francesco Mauri, Physica C 456, 38 (2007).

7 A. F. Hebard, M. J. Rosseinsky, R. C. Haddon, D. W. Murphy, S. H. larum, T. M. Palstra, A. P. Ramirez, and A. R. Kortan, Nature 350, 600 (1991).

8 J. Nagamatsu, N. Nakagawa, T. Muranaka, Y. Zenitani, and J. Akimitsu, Nature 410, 549 (2001).

9 J. Hlinka et al., Phys. Rev. B 76, 144512 (2007).

10 J. C. Chacon-Torres, A. Y. Ganin, M. J. Rosseinsky, and T. Pichler, Phys. Rev. B 86, 075406 (2012).

11 A. Marco Saitta, Michele Lazzeri, Matteo Calandra, and Francesco Mauri, Phys. Rev. Lett. 100, 226401 (2008).

12 I. P. Ipatova, A. V. Subashiev, Sov. Phys. JETP 39, 349 (1974).

13 E. G. Maksimov and S. V. Shulga, Solid State Commun. 97, 553 (1996).

14 I. P. Ipatova, A. V. Subashiev, and V. A. Shchukin, Fiz. Tverd. Tela 24, 3401 (1982); (Soviet Phys. Solid State 24, 1932 (1982)).

15 E. G. Maksimov, A. E. Karakozov, Physics-Uspekhi, 51, 535 (2008).

16 Yu. S. Ponosov, Sov. Phys. Solid State, 23, 861 (1981); Yu. S. Ponosov and G. A. Bolotin, Soviet Phys. Solid State, 27, 1581 (1985); Yu. S. Ponosov , G. A. Bolotin , G. P. Kovtun, and V. E. Elenskii, Sov. Phys. Solid State 26, 491 (1984).
17 Yu. S. Ponosov, I. Loa, V. E. Mogilenskikh, G. AB̉olotin, and K. Syassen, Phys.stat sol. (c) 1, 3114 (2004).

18 Yu. S. Ponosov, I. Loa, V. E. Mogilenskikh, and K. Syassen, Phys. Rev. B 71, 220301 (2005).

19 Yu. S. Ponosov, S. V. Streltsov and K. Syassen, High Pressure Research 32, 138 (2012).

20 Yu. S. Ponosov, G. A. Bolotin, C. Thomsen, and M. Cardona, Phys. Stat. Sol. (b) 208, 257 (1998).

21 J. R. Sandercock, Phys. Rev. Lett. 28 , 23740 (1972).

22 A. Dervisch and R. Loudon, J. Phys. C 9, L669 (1976).

${ }^{23} \mathrm{Yu}$. V. Knyazev and V. M. Maevsky, Optics and Spectroscopy 57, 253 (1984).

${ }^{24}$ V. V. Nemoshkalenko, V. N. Antonov, V. N. Antonov, M. M. Kirillova, A. E. Krasovskie, and L. V. Nomerovannaya, Sov. Phys. JETP 63, 115 (1986); V. N. Antonov, M. M. Kirillova, Y. Y. Krasovskiy, L. D. Kurmaeva, and N. V. Minulina, Phys. Met. Metallogr. 6, 78 (1990); M. M. Kirillova and L. V. Nomerovannaya, Sov. Phys. Solid State, 20, 568 (1978).

25 S. V. Shulga, O. V. Dolgov, and E. G. Maksimov, Phys. C 178, 266 (1991).

26 N. Wakabayashi, R. H. Sherm, and H. G. Smith, Phys. Rev. B 25, 5122 (1982).

27 R. Heid, L. Pintschovius, W. Reichardt and K. P. Bohnen, Phys. Rev. B 61, 12059 (2000)

28 O. K. Andersen and O. Jepsen, Phys. Rev. Lett. 53, 2571 (1984).

29 O. Jepsen, O. K. Andersen, and A. R. Mackintosh, Phys. Rev. B 12, 3084 (1975).

30 B. A. Sanborn, P. B. Allen, and D. A. Papaconstantopoulas, Phys. Rev. B 40, 6037 (1989).

31 P. P. Singh, Phys. Rev. B 75, 125101 (2007).

32 C. Stassis, D. Arch, B. N. Harmon, and N. Wakabayashi, Phys. Rev. B, 19, 181 (1979).

33 M. Cardona and I. P. Ipatova, in Elementary Excitations in Solids, edited by J. L. Birman, C. Sebenne and R. F. Wallis (Elsevier Science Publishers B.V., 1992), p. 238. 